\title{
Polysèmes
}

Revue d'études intertextuelles et intermédiales

\section{Le descriptif}

\section{Le descriptif désaffecté : The Enigma of Arrival de V.S. Naipaul}

Catherine Lanone

\section{(2) OpenEdition}

Journals

Édition électronique

URL : http://journals.openedition.org/polysemes/1746

DOI : 10.4000/polysemes. 1746

ISSN : 2496-4212

Éditeur

SAIT

Édition imprimée

Date de publication : 2 janvier 2007

Pagination : 55-69

ISSN : 0999-4203

\section{Référence électronique}

Catherine Lanone, «Le descriptif désaffecté : The Enigma of Arrival de V.S. Naipaul », Polysèmes [En

ligne], 9 | 2007, mis en ligne le 01 janvier 2007, consulté le 22 avril 2019. URL : http://

journals.openedition.org/polysemes/1746 ; DOI : 10.4000/polysemes.1746

Ce document a été généré automatiquement le 22 avril 2019.

Polysèmes 


\title{
Le descriptif désaffecté : The Enigma of Arrival de V.S. Naipaul
}

\author{
Catherine Lanone
}

Philippe Hamon définit le descriptif comme une série d'opérations en boucle (anaphore, paradigme, jeu sur le déjà-lu et le déjà-vu) déjouant la logique d'une diégèse qui serait, elle, toujours nécessairement orientée, vectorisée. Avant Hamon, on avait tendance à considérer la description comme un simple ornement, moment de stase où le récit se retrouvait au point mort. Si le concept de Hamon est toujours opératoire, c'est qu'il a su repérer, au contraire, une dynamique propre au descriptif, pour en faire une modalité de résistance à la diégèse : "L'essence du descriptif, s'il devait y en avoir une, son effet, serait dans un effort : un effort pour résister à la linéarité contraignante du texte, au post hoc ergo propter hoc des algorithmes narratifs $\|^{1}$. C'est peut-être la raison pour laquelle ce type d'écriture tient une place aussi importante dans la littérature post-coloniale, comme dans le cas de The Enigma of Arrivai de V.S. Naipaul, livre qui défie toute logique diégétique pour explorer à la place les plis du descriptif, de la peinture de paysage à l'hypotypose ou à la légende d'un tableau. Les descriptions déclinent ainsi le double paradigme du déplacement et de la stase, pour réinventer le topos du récit de voyage, comme le rappelle Tobias Döring: "While all the autobiographical narratives may rely on the symbolic topography of travel by virtue of the life-as-journey metaphor, Caribbean autobiographers of postcolonial predicaments newly invest such metaphors with political significance derived from their experience of migration $»^{2}$. Le descriptif révèle l'envers $d u$ décor, problématisant le cadre idéologique qui sous-tend la perception.

Qualifié sur la couverture de "roman », The Enigma of Arrivai surprend d'emblée le lecteur en proposant un parcours autobiographique où la description l'emporte délibérément sur la narration. On ne trouve plus guère ici ces modes de démarcation recensés par Hamon, visant à cadrer les descriptions dans un roman ; la description, ici, gagne en ampleur et se poursuit sur quelque trois cents pages. Le livre tient du poème en prose, saturé d'échos, de répétitions, au gré de rythmes anaphoriques lancinants. Les dialogues sont distillés sous forme de bribes, la diégèse s'efface, ou presque ; les événements les plus marquants 
(une mort, un crime passionnel) s'apprennent au détour d'une phrase ; au contraire, les descriptions - d'un costume, d'une activité liée au jardinage, d'un lieu, parfois (mais rarement) un intérieur cadré par l'embrasure d'une porte, le plus souvent un chemin, un paysage, un parcours -, se travaillent, se reprennent, se déploient au fil du texte, comme s'il s'agissait, précisément, de tisser des impressions, et cent fois sur le métier de remettre l'ouvrage.

3 Sommé d'être attentif aux redites, à la répétition obsédante mais aussi aux légers écarts qui permettent d'assembler les éléments sporadiquement présentés, le lecteur est donc convié à parcourir l'espace du texte, comme s'il partageait les promenades du narrateur. Les deux chemins qui guident vers la petite maison de Jack fonctionnent comme indice métatextuel : au tracé rectiligne de l'asphalte moderne, le narrateur préfère les méandres d'un chemin issu d'un cours d'eau à sec, voie sinueuse qui correspond à ce récit décentré, excentrique, délibérément lent et répétitif, qui ramène toujours à Jack. Au départ, lorsqu'il apparaît dans le texte, le jalon (le jardin de Jack, le cottage de Jack) joue sur l'asémanthème du nom propre, comme si le lecteur connaissait déjà ce Jack qui n'apparaissait que dans le titre du chapitre. Le repère reparaît en point d'orgue, à la dernière phrase du livre, point de départ et point d'aboutissement de toute description et du récit même que nous venons de lire : «I laid aside my drafts and hesitations and began to write very fast about Jack and his garden $»^{3}$. De fait, la description se cultive, à la manière de ce jardin de Jack posé d'emblée comme la matrice de l'écriture (" this ability [...] to enter my writing as I might enter a walled garden or enclosure », 154).

4 Par le biais de la reprise, soit allusive soit au contraire travaillée, amplifiée, le paysage fonctionne alors comme palimpseste, trace d'un monde intérieur ( «Land partakes of what we breathe into it, it is touched by our moods and memories ", 301) mais aussi feuilletage temporel par surimpression des sensations et des saisons. Ainsi, les printemps se fondent devant le miroir d'eau impressionniste qui reflète les arbres et les plantes «succulentes»:

[...] after that first spring I would say: "at least I had a spring here". And then I said:

"at least, I had a spring and summer here". And : "At least I've had a year here".

And so it went on, as the years passed. Until time began to telescope and experience

began to change: the new seasons not truly new any more, bringing less of new

experience than reminders of the old. (82)

5 Michel Beaujour définit l'écriture autobiographique comme un parcours où le promeneur dépose des images dans les lieux, comme s'il les gravait sur une tablette de cire, afin de pouvoir un jour les y retrouver, les reprendre et les relire ${ }^{4}$. De même, le parc ne cesse de se relire; l'image du narrateur, écrivant dans son cottage, et celle du "livre d'heures " composé par les rituels de Jack le jardinier, se répondent.

Ce parcours mnémonique s'inscrit dans une tradition à la fois littéraire et picturale. La solitude nostalgique évoque le romantisme. À mesure que le lieu guérit d'atroces migraines et cauchemars, le narrateur ressent un épiphanique sentiment de renaissance. Agés, courbés, le beau-père de Jack ou le père de Mr. Phillips font office de présences tutélaires, lointains descendants du « leech gatherer » de Wordsworth. De même que l'on ne voyait jamais Tintern Abbey dans le poème éponyme de Wordsworth, Naipaul ne guide jamais son lecteur jusqu'à Stonehenge, inaccessible point de fuite du récit. Mais l'enchantement convoque aussi le modèle pictural. La description décline les topoi d'un paysage à la Constable, avec ses aubépines en bordure de sentier, ses meules de foin dorées, la barrière sur le chemin, l'éblouissement d'une pivoine ou d'un iris, l'intimité du 
cottage auquel on revient toujours: "And always there was the river, with its overwhelming beauty of reeds and weeds and changing reflections » (189). La promenade se compose, comme une série d'esquisses ou d'études au trait allusif ou au contraire appuyé, série d'échos changeants : «Jack's garden, ivy, rooks; the old rose-bed (without roses now, a briar wilderness, long after that first summer of thorny, dense-petalled, scented, lilac-pink roses)» (191). Pour Lucian Freud, toute l'émotion chez Constable naît du détail, d'un simple chemin qui conduit à une barrière, comme si soudain tout était là, dans cette barrière ${ }^{5}$. Comme Constable, qui voyait dans chaque haie, chaque chemin l'essence même de son art, Naipaul s'attache à peindre le détail du paysage, autant que le palimpseste des saisons et des impressions. C'est que la promenade semble mener au cœur même de la vue, et donc d'un tableau qui s'animerait :

Just in this way now the water meadows had the effect (in one corner of the mind) of abolishing the distance between Constable and the present: the writer, the man with his colours and brushes and boards, seemed as near and as contemporary as what he made us now see: the water channels and the pollarded willows he had settled down one day to paint. This idea of the painter, this glimpse of the painter's view, made the past ordinary. The past was like something one could stretch out and reach; it was like something physically before one, like something one could walk in. (170)

7 La répétition de "this » impliquant une proximité, de l'analogie "like something ", fondent par surimpression le paysage peint et le paysage réel, mêlant les temporalités.

Le rapport spéculaire écriture-peinture fait donc miroiter le thème de l'enchantement, de la renaissance romantique dans la matrice spatiale du Wiltshire. Mais la magie du paysage ne se donne pas d'emblée, elle se gagne, se conquiert. Dans un premier temps, l'initiation herméneutique paraît épiphanique. Il faut apprendre à déchiffrer l'espace, la connaissance distillée par le temps passé dans le Wiltshire. Si le modèle pictural ou littéraire donne des clefs, il faut aussi se familiariser avec l'espace, seuls la promenade et le temps peuvent permettre de le connaître intimement. La promenade devient donc un rite d'apprentissage. Au départ, le narrateur affiche une connaissance théorique, géographique, culturelle et linguistique; il sait, par exemple, que le signifiant tautologique Waldenshaw veut dire deux fois « wood». Mais il lui faut acquérir un autre type de compétence pour pouvoir lire correctement le paysage. Le roman s'ouvre sur l'énigme de l'arrivée, une carte blanche illisible noyée de pluie : « For the first four days it rained. I could hardly see where I was » (11). Puis les jalons apparaissent peu à peu, comme le ferait une écriture à l'encre sympathique; le narrateur aperçoit quelques arbres, des champs, les reflets d'une rivière qu'on appelle l'Avon, mais qui n'est pas l'Avon de Shakespeare. Cet incipit crée une nouvelle Genèse, laissant émerger peu à peu le paysage du voile de pluie, comme s'il se créait à mesure que le narrateur en discerne les éléments constitutifs. La description est scandée par la répétition de "I saw", psalmodiant sur le mode de l'incantation obsédante ce premier éveil de l'œil au paysage. Lorsque la neige gomme à son tour la plupart des traits du lieu, la structure intime de la vue se laisse paradoxalement appréhender, tableau abstrait d'une terra incognita qui s'offre au voyageur de l'intérieur, découvrant l'Angleterre comme un étrange ailleurs, inversant le regard porté par les conquérants de jadis. Puis la promenade, le fait d'arpenter inlassablement l'espace, l'apprivoise, le rend plus familier, avec ses motifs obsédants, topoï du récit, comme le parc avec ses lapins ou ses faons, ou les serres à demiruinées et les roses du cottage, les arbres majestueux ou déchus, coupés. Mais ce n'est que peu à peu que les vues s'agencent pour faire sens : «I saw what I saw very clearly. But I 
didn't know what I was looking at. I had nothing to fit it into. I was still in a kind of limbo» (12). Seul le rite d'initiation au lieu, avec une infinie patience, peut dissiper les limbes.

9 Il faut donc apprendre à discerner le paysage et l'empreinte du temps, à lire le costume du jardinier comme un Livre d'Heures, à s'initier au rythme des floraisons, des saisons et des habitudes, opérer une sélection dans les impressions enchevêtrées, comme on apprend une langue étrangère. Le texte reprend sans cesse ce motif de l'apprentissage d'un savoir :

I saw with the eyes of pleasure. But knowledge came slowly to me. It was not like the instinctive knowledge that had come to me as a child of the plants and flowers of Trinidad: it was like learning a second language. (32)

10 L'amour du lieu s'éveille, tisse une magie, un enchantement au fur et à mesure que le narrateur apprend qu'un jardin se cultive, qu'il faut susciter mais aussi contrôler la vie végétale. Le jardin de Jack n'existe que parce que Jack y travaille sans relâche, le parc reste en suspens, grâce aux efforts de Pitton, seul rescapé des seize jardiniers de jadis, luttant pour limiter la déchéance.

11 Une autre leçon se glisse alors dans l'éveil des sens et de l'âme aux lieux. Même si la nature vient guérir le narrateur d'angoisses et d'atroces maux de tête, le jardin n'est ni Eden ni Arcadie, il n'appartient pas à l'éternel âge d'or. Pourtant, longtemps, le narrateur a le sentiment d'être parvenu à pénétrer l'essence même de l'Angleterre, comme si le temps s'y distillait, y suspendait son vol, comme si le paysage n'avait pas changé depuis Constable, voire depuis toujours. Le narrateur décline ce sentiment d'appartenance ancestrale : "Of literature and antiquity and the landscape Jack and his garden and his geese and his cottage and his father-in-law seemed emanations » (25). Mais le sortilège est trompeur. La perception intemporelle qui mêle sacré et profane n'est qu'illusion, et pas seulement parce que ce sont peu à peu les signes de la mort en ce jardin, de la déchéance et de la finitude, qui s'imposent. La mort de Jack, les départs ou décès successifs, entraînent une série de dégradations de l'espace qui minent le modèle immuable hérité de Constable ou Wordsworth. La référence à un modèle pictural ou littéraire pose le problème d'une norme descriptive canonique, qu'il faut à son tour repérer et déchiffrer.

12 À mesure que s'affirme la célébration du lieu, et sans jamais s'en départir, un courant ironique vient déconstruire systématiquement l'approche mythique intemporelle. Car l'espace qui se donne à lire comme mythe est toujours détourné, faussé, il porte le poids d'une construction idéologique qui vient subvertir la poétique de l'espace: la vision relève toujours du préconstruit culturel. À la manière des promeneurs anglais cultivés contemplant jadis le paysage avec un miroir de poche, le miroir de Claude visant à métamorphoser toute vue en tableau du Lorrain, le narrateur se rend compte que sa perception est filtrée par une grille culturelle, un prisme déformant, toujours plaqué sur le paysage. D'emblée, nous disait le texte en une formule à la Magritte, cette rivière n'est pas l'Avon.

13 Le titre nous invite à une lecture herméneutique. L'énigme, c'est le signifiant en quête de signifié, mais résoudre l'énigme, ce n'est pas seulement apprendre à lire l'espace, c'est se déprendre du cliché, de l'illusion prégnante de l'allusion.

14 Car l'intemporalité projetée sur l'Angleterre, c'est l'envers de l'orientalisme. En fait, l'espace inlassablement parcouru est soumis au temps : il est peu à peu coupé de clôtures et de barbelés, jalonné de traces, comme ces ruines de bâtiments que l'on abandonne, ou 
ces meules d'or qui pourrissent, ou encore ces pneus au milieu desquels jouent les enfants. Le tas de feuilles et de résidus à brûler devient "refuge » au lieu de "refuse ", par un léger glissement connotant, sous l'aspect rassurant, la déchéance de cette matrice mortifère.

Le jeu sur les mots pourrait faire croire que la ruine s'inscrit dans le paysage, pour le rendre pittoresque. Or, il s'agit d'un processus de lamination, qui implique de réviser radicalement la description : l'espace perçu comprend plus ostensiblement un champ de tir militaire que le cercle sacré de Stonehenge. La leçon de lecture se poursuit, sur le mode dysphorique. Le paysage initialement indéchiffrable, marqué au sceau d'une cécité qui progressivement se dissipe, réécrit ironiquement le motif de la mort dans le jardin, «Et in Arcadia Ego ». Le « hortus conclusus » tient du fantasme issu de la lecture, une construction culturelle plus qu'une réalité :

So much of this I saw with the literary eye, or with the aid of literature. A stranger here, with the nerves of the stranger, and yet with a knowledge of the language and the history of the language and the writing, I could find a special kind of past in what I saw; with a part of my mind I could admit to fantasy. (22)

Il faut sans cesse réinscrire le lieu dans la temporalité. Le narrateur doit donc se déprendre de l'exaltation pour acquérir une vraie compétence, il doit non seulement apprendre à lire le paysage mais aussi à se détacher des stéréotypes culturels. Il faut repérer et se défier de cet «horizon d'attente prétabli » qui régit selon Hamon perception et description, « donc, avec une norme, un code, un modèle préexistant " ${ }^{6}$.

Décrire revient alors à identifier les leurres qui voilent la perception. Ainsi, le couple ancestral de domestiques veillant sur le manoir, les Phillips, vient en fait d'arriver dans la vallée. Il faut repérer les dates gravées sur les constructions, comme ce 1911 qui fait d'un «cottage» le simulacre d'un modèle à la Constable, un agencement idéologique. Il convient de s'émanciper du calque culturel pour pouvoir écrire la carte du lieu.

La description devient donc le lieu d'un enjeu métatextuel, c'est tout le devenir de l'écrivain qui s'y joue. La promenade, qui semblait la clef d'un espace intemporel, se doit d'être recontextualisée, historicisée. L'exilé venu de Trinidad se sent chez lui dans ce Wiltshire désenchanté où il joue également le rôle de l'intrus. Car métonymiquement, la splendeur passée du parc, en marge duquel se trouve le petit cottage où loge l'écrivain, renvoie à une splendeur passée qui s'inscrivait jadis, nécessairement, dans une économie coloniale. Le narrateur voit dans son propriétaire un double inversé, dont le rapproche et le sépare, littéralement, un empire.

19 S'initiant au paysage, l'œil doit aussi se laver d'un rêve de perfection entaché de passivité. Le trope de l'aveuglement est ironiquement mis en scène lorsque l'écrivain aperçoit le propriétaire qui passe en voiture, et note avec gratitude l'étincelle bienveillante dans le regard, le petit signe de la main. Or, Mr Phillips lui explique par la suite que le propriétaire est à demi-aveugle et porte des lunettes noires. Les regards ne se croisent donc jamais, la perception était biaisée par l'idée reçue, la description de la rencontre sincère mais faussée. Le narrateur comprend qu'il faut avant tout s'émanciper du fantasme colonial de l'anglicité pour dessiller le regard.

Déroulant ses volutes, reprenant ses topoi, le texte représente le parcours des promenades tout en introduisant du jeu dans la description pour exalter le lieu mais aussi saper le mythe. Ainsi, le narrateur rappelle que le paysage aux vaches vient bien de Constable, mais aussi d'une autre image, celle de l'étiquette de la boîte de lait condensé qui fascinait l'enfant de Trinidad. La perception est faussée par le cliché, signifiant prêt à consommer 
absorbé jadis comme une deuxième langue maternelle. L'étiquette fonctionne comme mythème au sens de Barthes, comme talisman idéologique, comme ces livres publiés en temps de guerre, ces volumes Penguin brochés, aux agrafes tout de suite rouillées par le climat tropical, mais dont le papier fleure bon les rêves dans la mémoire du narrateur. objet sensuel, le livre est aussi un objet de consommation véhiculant, comme idéologie, les clichés d'une inaccessible Angleterre, signifiants connotant implicitement l'indiscutable présence coloniale. Mais la guerre creuse déjà une brèche dans le système : la publicité sur la couverture vante parfois un produit qui n'est plus fabriqué en cette période trouble, talisman performatif destiné à garantir la réapparition du produit une fois la guerre terminée. Le faux-monnayage signifiant continue de vendre, avant tout, du rêve aux colonies : " these advertisements - for things doubly and trebly removed from possibility - never struck me as odd: they came to me as an aspect of the romance of the world I was working towards, a promise within the promise, and intensely romantic " (121).

21 C'est cette romance coloniale que le narrateur se doit de mettre en perspective, comme le rappelle Döring : «For his visual observations, central though they may be to articulate his subjectivity, turn out to be dominated by pre-fabricated views, images long familiar from colonial circulation ${ }^{7}$. Il lui faut se dégager du modèle littéraire, qui le hante lorsqu'il arrive à Londres et s'imagine que la métropole va correspondre au Londres de Dickens réinventé, transposé par l'enfant de Trinidad avide d'ailleurs et de lecture. Les trous du «Blitz» semblent signer la béance qui se creuse entre fantasme et réalité. L'énigme de l'arrivée, c'est celle qui inverse le parcours colonial, pour implanter dans l'enclave métonymique du Wiltshire l'écrivain venu de Trinidad, coupé de sa terre natale, hanté par des clichés dont il doit se déprendre.

C'est alors qu'intervient dans le texte la description par excellence, non plus l'hypotypose dans le style de Constable, mais l'ekphrasis, la mise en abyme d'un tableau cité puis décrit. Mais il ne s'agit plus de Constable, le tableau vient s'inscrire à l'ouverture de la deuxième partie en complet décalage avec l'horizon d'attente construit dans la première partie. Le paradigme de la référence spéculaire se révèle être le tableau éponyme de Giorgio de Chirico, L'Enigme de l'arrivée 8 . Le changement de style fait déraper la lecture, puisque non seulement la description est enchâssée, mais le titre se dissémine, sert de motif structurel au récit autobiographique.

D'ordinaire, devant l'ekphrasis, le lecteur a plutôt tendance à éprouver un sentiment de plénitude, à la suture du lisible et du visible. Voilà la peinture assignée dans le texte, pour y faire jaillir un entre-deux figural dans le pli du langage. Selon Bernard Vouilloux, "l'ordre du décrit s'ouvrirait au récit du regard, l'inventaire à l'événement scopique »" Mais l'insertion du tableau, ici, est à la fois limpide et malaisée. Le style de De Chirico s'intégre mal dans le parcours pictural à la Constable. L'image crée un hiatus, une brèche, le rythme se syncope à l'ouverture de cette deuxième section où le lecteur s'imaginait invité à poursuivre sa promenade en marge des jardins, et se retrouve au contraire expulsé dans l'espace lisse et froid de ce quai énigmatique représenté par De Chirico. Comme pour mieux simuler le dispositif de mise en scène textuelle qui se joue dans l'ekphrasis, le narrateur ne décrit d'ailleurs pas le tableau, mais une simple reproduction, tirée d'un petit livre banal trouvé par hasard dans le cottage. En fin de compte, le tableau n'est pas ici évoqué mais convoqué, comme une présence qui viendrait conjurer l'angoisse en lui donnant forme. La description de la toile ne vient pas satisfaire une attente du lecteur par un surplus de jouissance visuelle, elle vient au contraire donner 
forme à un malaise, une absence, provoquer une parole au moment où la brume d'humidité noyant le paysage concrétise l'aphasie guettant l'écrivain déchiré par l'exil, errant dans les limbes de l'hybridité culturelle :

Au tableau, dit-on, il ne manque que la parole: n'est-ce pas une manière de souligner ce qui fait du tableau un interlocuteur potentiel, l'autre silencieux qui me provoque à parler - qui me provoque ? Le tableau ne me parle pas, mais il s'adresse à moi dans l'idiome inouï qui est le sien (il me regarde) et il m'incite à parler - à parler au tableau (ou à l'une de ses figures) où à parler de lui à un tiers. ${ }^{10}$

Le tableau « incite à parler ", provoque une parole, catalyse la description, ou plutôt des légendes descriptives. On a d'une part l'ekphrasis, le discours qui articule le tableau, permet au lecteur de se le représenter, de s'en bricoler, dit Vouilloux, «une représentation mentale, de s'en faire une image ». Simultanément, le tableau inspire à Naipaul un autre type de récit, un voyage imaginaire dans l'antiquité qui viendrait expliquer la posture énigmatique des deux personnages peints sur la toile, ainsi que cette voile qui s'éloigne, derrière le mur qui ferme la perspective. Ce récit qui copie la toile ne s'écrira jamais vraiment, mais l'impulsion reste l'étincelle qui permet au texte que nous lisons de s'écrire, beaucoup plus tard. Décrire le tableau, c'est pour Vouilloux restituer le tableau dans sa présence,

[...] c'est-à-dire essentiellement cette avancée (praesens) qui a fait de moi, spectateur, son destinataire et par où il vient à me toucher; décrivant le tableau, c'est cette pointe sensible, ce contact - localisable peut-être dans ce que Barthes appellerait le punctum (ce qui me point) du tableau - dont il m'est loisible de rendre le frémissement. ${ }^{11}$

La toile est une présence qui vient littéralement toucher le narrateur, par la piqûre de l'affect. Le tableau de De Chirico est ce point névralgique qui se déplie en paradigme, en scénario programmatique. Désarrimé (le narrateur est toujours surpris lorsqu'il revoit la reproduction de l'original, qui ne correspond jamais à son souvenir), pris dans la mouvance du parcours intérieur, spatial, textuel, le tableau devient à son tour un objet fantasmatique, mais il ne s'agit plus d'un fantasme imposé idéologiquement par l'empire britannique ; la vision romancée de l'Antiquité guide vers l'écriture d'un roman situé en Afrique, puis vers ce récit autobiographique atypique qu'est The Enigma of Arrival.

En ce sens, la description du tableau devient moins importante que le descriptif, au sens propre, c'est-à-dire le titre même du tableau, qui fonctionne comme une légende, une clef de lecture, titre que Naipaul emprunte à De Chirico, qui l'avait lui-même emprunté à Apollinaire. C'est le titre qui donne au tableau toute sa valeur, parce qu'il exprime tout l'affect de l'exil : "The scene is of desolation and mystery: it speaks of the mystery of arrival. It spoke to me of that, as it had spoken to Apollinaire» (91-92). Apposé à la reproduction du tableau, sur la couverture, le terme de "roman » propose un jeu de pistes à la Magritte : ceci (n')est (pas) une autobiographie. L'énigme de l'arrivée, c'est l'énigme du destin, du devenir-écrivain, paysage intérieur autant que tableau du déclin de l'empire colonial.

27 C'est pourquoi la mise en abyme décalée du tableau ébranle les modalités de description très nostalgiques de la première partie. Elle ouvre une section intitulée "The Journey ", où se rejoue le scénario de l'incompréhensible arrivée, une première fois dans le Londres scarifié par la guerre, une deuxième fois dans le Wiltshire, après que Naipaul avait cru pouvoir quitter pour de bon l'Angleterre, avant de se voir contraint de revenir parce que l'éditeur avait refusé de publier le livre de voyage qui devait permettre de financer un nouveau départ, à Trinidad. Le « roman » qui naît du Wiltshire s'interroge sur ce parcours 
en réinventant la description et ses modalités, pour témoigner de ce devenir-écrivain dans un lieu qui dessille le regard, et donne donc accès à un nouveau type d'écriture, fructueuse et inattendue.

On pourrait résumer la dialectique de l'exil et de l'appartenance, entre description fluide et aphasie, par un carré sémiotique : au départ, le narrateur veut voir l'Angleterre, et pour cela, il refuse de voir sa propre culture (il ne veut pas voir tout ce qui se rapporte à Trinidad, à son statut de voyageur de couleur, et note nombre d'anecdotes insignifiantes pour copier le récit trop blanc qu'il s'imagine devoir écrire). Une fois dans le Wiltshire, il veut ne pas voir certains signes de déchéance ou d'indifférence de la part du propriétaire du manoir, pour couler l'expérience dans le moule d'un fantasme idyllique. Il apprend peu à peu, au fil des promenades et du texte, à ne pas vouloir ne pas voir, à intégrer dans son récit l'expérience de l'éphémère, de la déchéance, de la démystification. L'écriture se place alors sous le signe de l'intégrité scopique, un désir de voir au plus près le paysage anglais, dans son ambivalence, sa grâce cathartique et sa puissance mortifère, et l'identité, tout aussi incertaine, du narrateur lui-même :

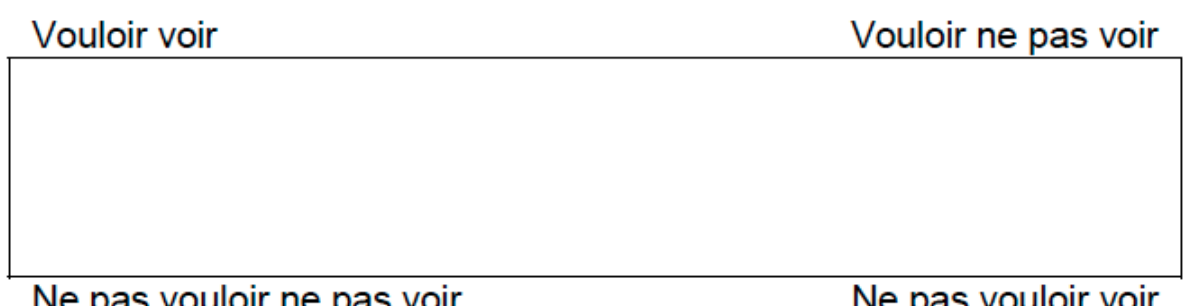

29 Vouloir voir, c'est donc aussi vouloir écrire, s'émanciper des clichés, ou plutôt les subvertir, les écouter et les dérouter, se les approprier pour les détourner, au fil de cet étrange parcours en boucle.

Ainsi, les topoi naïfs se dégradent : la maison d'enfants, abandonnée, a été entourée de barbelés, par crainte des vagabonds ; la paille filée d'or pourrit ; on trouve bien un jardin secret, avec sa porte dérobée tout droit sortie du Secret Garden de Frances Hodgson Burnett, avec ses arbres en fleurs et sa fontaine aux carreaux bleus; on le découvre, on le nettoie, et puis on le referme et on l'oublie, car l'on ne sait qu'en faire. Le lieu se replie sur le vide, mélancolique, marqué au sceau de la maladie de langueur du propriétaire.

Peu à peu, le texte renvoie à l'Angleterre un miroir ironique où la progression enchantée s'inverse en voyage vers une inextricable jungle. Si la description, supplantant toute narration, semble emprunter un cours sinueux semblable à ce lit de rivière asséché, le parc est jonché d'arbres abattus, de ponts pourrissants, symboles d'un lieu colonial qui s'est défait. Le lierre déploie ses vrilles signifiantes, ses rets étouffants, dans la section éponyme où le motif se décline de façon envoûtante. Or, c'est le propriétaire qui a demandé qu'on ne coupât jamais le lierre: "What were his feelings, then, when a tree collapsed? So many trees had collapsed. Such a wilderness now ruled the water meadows, such a forest litter» (194). Le lierre gagne, ronge et étouffe, le lieu est miné par la paralysie, la corruption, la stase fétide, comme si l'acédie du propriétaire, sa maladie de l'âme, contaminait l'espace. Le dernier jardinier qui lutte pour maintenir l'ordre, Pitton, est renvoyé, cédant la place aux intérimaires, avec leur futile dépense d'énergie sporadique incapable d'endiguer le déclin. Ce n'est pas un hasard si le narrateur affirme ne pas trouver «creepy» ce propriétaire qu'il ne voit jamais. A contrario, le signifiant 
s'immisce pour nouer le propriétaire devenu parasite en son domaine et les lianes du lierre.

Cette langueur mortifère métamorphose la description, lui insuffle une puissance envoûtante. La jungle gagne, dans l'isotopie des herbes folles creusant le paysage de zones d'ombre, de profondeurs inquiétantes. L'espace, sauvage, semble changer d'échelle. L'eau noirâtre gagne insidieusement, les structures s'effondrent, comme ces maisons de verre que sont les serres: "Bush inside the great greenhouse, bush outside» (186). La luxuriance tropicale, que l'on voulait contenir dans la serre exotique, se dissémine, tandis que la référence isotopique se précise, auprès de la «ruine spectaculaire » (187) du hangar à bateau :

But tame though the river looked - a few feet deep, a few feet wide - the water it channelled was a force of Nature, not wholly predictable; and the banks of that river and its inlets were constantly changing. The boathouse creek, widening or simply shifting, had caused the boat to collapse on one side. The angle of collapse, the rotting timber, the black-looking water, the rusting corrugated iron suggested a tropical river ruin, somewhere on the Orinoco or Amazon or the Congo. (188)

Le pantonyme apparaît implicitement avec la référence au Congo : comme le souligne Döring, ce qui se joue implicitement, ici, c'est un voyage au cœur des ténèbres à l'envers, puisque la démesure luxuriante se situe cette fois en Angleterre. Le propriétaire, entrevu les jambes croisées, chauve dans un rayon de soleil, évoque un Kurtz affadi et parodique, voué au mutisme. De même, le manoir est comparé à un navire à vapeur, et ce n'est pas un hasard si une chaudière y explose, évoquant là encore un récit de Conrad. Le mythe du jardin, de l'anglicité idyllique, s'effondre, rongé par ces puissances délétères jadis attribuées à l'ailleurs. Dans cette perspective, le parc n'est plus qu'une «utopie dégénérée ", " une idéologie réalisée sous la forme d'un mythe » pour reprendre le mot de Louis Marin ${ }^{12}$.

Le narrateur se rend compte que même s'ils partagent, sans se croiser, le même espace, le propriétaire et son locataire ne sauraient avoir la même perspective, ils ne sauraient littéralement voir la même chose.

Dans ce voyage au cœur des ténèbres qui s'inverse (le narrateur n'écrit-il pas un roman sur l'Afrique à son arrivée dans le Wiltshire ?), un autre type d'écriture s'ébauche, tissant ou métissant les images pour désancrer les clichés culturels, y introduire du jeu. Ainsi, la neige et le sable se confondent, et lorsque les roseaux se disséminent après le départ de Pitton, le narrateur évoque un embrasement tropical : « [they] seeded themselves in the lawn - like the trash from a sugar-cane fire jumping a fire-break and sending arrows of flame into the adjoining green field» (184). À l'inverse, le bruit d'un incendie lorsque prend feu une pinède rappelle une chute d'eau de la jungle, " giving me the idea that all matter was one, and all disturbances, whether fire or water or air, were the same » (297). C'est le deuil d'une culture, du moins sous sa forme hégémonique, qui permet la renaissance de l'écrivain.

Le parcours intime réécrit alors le traditionnel motif d'« Et in Arcadia Ego ». Le bâtiment à l'arrière-plan du tableau de De Chirico en gardait peut-être une trace, évoquant la tolos funéraire de certains paysages romantiques. Dans The Enigma of Arrival, parcours au sein des vestiges du jour, les sections s'articulent discrètement autour de la mort, non seulement la déchéance du lieu mais aussi le décès de Jack, de Mr Phillips, de la sœur du narrateur. La mort s'immisce par déplacement, c'est le jardin en friche qui signe la mort de Jack, l'adjectif se glisse au détour d'une description, «the dead cottage of Jack » (46), 
pour révéler l'absence, la déchirure de la trame spatiale lorsque disparaît le patient jardinier. La mort se produit hors du récit, seule reste cette lente méditation, ce ressassement au fil des promenades nostalgiques, cette mélancolique traversée entre deuil et renaissance. C'est alors qu'il faut, en boucle, se souvenir de la dédicace qui ouvrait le livre, et de la mort que le récit passe sous silence, celle du frère, qui se produit au cœur de la rédaction. C'est le silence du texte, comme un soupir au sens musical du terme, qui crée la résonance de l'énigmatique parcours dans l'espace désaffecté du Wiltshire.

Pour conclure, la description n'est pas une modalité figée, cadrée dans The Enigma of Arrival, mais un devenir, toujours pris au milieu et qui se déploie sans cesse. La peinture du lieu, espace d'une réflexion métatextuelle sur l'écriture et la représentation, dénoue la dialectique coloniale du centre et de la marge (la promenade en bordure d'espace s'articule autour de la figure de Jack, qui, nous dit le narrateur, reste à la périphérie de sa vie à lui), là où l'arrivée rejoue toujours l'énigme du commencement, qu'il s'agisse du lieu ou de l'écriture: "for several weeks I made many starts, allowing my hand to run; starting at different points » (310). Il ne s'agit donc pas de brûler tout l'héritage culturel, de Wordsworth à Constable, mais de se déprendre de l'illusion mimétique, de la perception romantique, pour historiciser la description. Le palimpseste descriptif confronte les empreintes culturelles, les métisse, pour cerner, dans la répétition à l'excès, l'irreprésentable, les énigmes de l'arrivée, l'écriture, le lieu, la mort: "I went for my walks every afternoon. I finished my book » (96).

\section{NOTES}

1. Philippe Hamon, Introduction à l'analyse du descriptif, Paris : Hachette, 1981, 5.

2. Tobias Döring, Caribbean-English Passages: Intertextuality in a Post-Colonial Tradition, London: Routledge, 2002, 213.

3. V.S. Naipaul, The Enigma of Arrival, Harmondsworth: Penguin, 1987, 318.

4. Michel Beaujour, Miroirs d'encre, Paris : Éditions du Seuil, 1980.

5. Voir John Gage, Anne Lyles, Constable: le choix de Lucian Freud, Paris: Réunion des musées nationaux, 2002.

6. Philippe Hamon, Introduction à l'analyse du descriptif, op. cit., 127. Pour Hamon, la description fonctionne toujours comme opérateur d'intertextualité: «describere, rappelons-le, étymologiquement, c'est écrire d'après un modèle » (51).

7. Tobias Döring, Caribbean-English Passages, op. cit., 127.

8. Giorgio de Chirico, The Enigma of Arrival and the Afternoon, 1911-1912, huile sur toile, $70 \times 86 \mathrm{~cm}$, collection privée.

9. Bernard Vouilloux, La Peinture dans le texte, Paris : CNRS, 1994, 115- 116.

10. Ibid., 116.

11. Ibid.

12. Louis Marin, Utopiques : jeux d'espace, Paris : Éditions de Minuit, 1973, 297. 
INDEX

oeuvrecitee Enigma of Arrival (The) 